\title{
The magic of nanoplasmonics: from superhydrophobic and 3D suspended devices for SERS/TERS-like applications to hot-electrons based nanoscopy
}

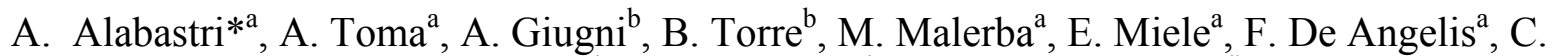 \\ Liberale $^{\mathrm{a}}$, G. Das ${ }^{\mathrm{b}}$, E. Di Fabrizio ${ }^{\mathrm{b}}$, R. Proietti Zaccaria ${ }^{\# \mathrm{a}}$ \\ astituto Italiano di Tecnologia (IIT), 30 Via Morego, Genova, 16163, Italy \\ ${ }^{\mathrm{b}}$ King Abdullah Univ. of Science and Technology (KAUST), Thuwal, Saudi Arabia
}

\begin{abstract}
The ability to confine light in small volumes, associated to low background signals, is an important technological achievement for a number of disciplines such as biology or electronics. In fact, decoupling the source position from the sample area allows an unprecedented sensitivity which can be exploited in different systems. The most direct implications are however related to either Surface Enhanced Raman Scattering (SERS) or Tip Enhanced Raman Scattering (TERS). Furthermore, while the combination with super-hydrophobic patterns can overcome the typical diffusion limit of sensors, focused surface plasmons decaying into hot electrons can be exploited to study the electronic properties of the sample by means of a Schottky junction. Within this paper these techniques will be briefly described and the key role played by both surface and localized plasmons will be highlighted.
\end{abstract}

Keywords: Surface Plasmon Polaritons, Adiabatic compression, Super-hydrophobic surfaces, SERS applications

\section{INTRODUCTION}

Diffraction limit, which sets a boundary in the optical maximal resolution, represents one of the deepest constrains for nanoscale optical sensing applications. One of the methods to overcome this limit is to consider electromagnetic waves bounded to metallic nanostructures, i.e. when their plasmonic properties are utilized [1-3]. Since the electric field of plasmonic oscillations decay directly from the structures, under proper excitation an optical signal can be brought down to nanometric spots therefore overcoming the conventional diffraction limit.

Plasmons are collective electronic oscillations which can be induced in metallic-like dispersive materials. Depending on the type of excitation, coupling conditions and geometry we can refer either to bulk plasmons, surface plasmons (SPs) or localized plasmons (LPs). Bulk plasmons are longitudinal oscillations at the plasma frequency of the bulk material, which for noble metals usually lies in the UV region. SPs are electromagnetic waves propagating along the interface between a dielectric and a conductor, evanescently decaying in both media. Finally, LPs are non-propagating waves which can be induced in metallic nanostructures [4]. In particular, they are characterized by the oscillation of surface electrons which are resonant at certain frequencies, hence leading to an electric near-field enhancement $[4,5]$.

When SPs properties are considered on a conical metallic nanostructure, an interesting phenomenon termed adiabatic compression rises [6]. On this phenomenon rely some of the applications which are going to be presented here. In particular, after briefly introducing adiabatic compression, its application to SERS/TERS and the combination to superhydrophobic surfaces or Schottky junction will be discussed.

\subsection{The adiabatic compression}

Babadjanyan et al. in 1999 [7] and M. Stockman in 2004 [8] theoretically showed how the wavelength associated to a surface plasmon propagating on an ideal metallic conical structure would tend to the zero, together with its group velocity, by approaching the cone apex. In such a condition high electric fields at the end of the tip were predicted, thus paving the way for new optical tools for the optical investigation of nanostructures $[6,9]$.

*alessandro.alabastri@iit.it; ${ }^{*}$ remo.proietti@iit.it; www.iit.it

Nanophotonics V, edited by David L. Andrews, Jean-Michel Nunzi, Andreas Ostendorf, Proc. of SPIE Vol. 9126, 91260A - @ 2014 SPIE · CCC code: 0277-786X/14/\$18 · doi: 10.1117/12.2052569 
An easy and intuitive way to understand this phenomenon is by considering a cone as a sequence of coaxial cylinders with diminishing radius. Furthermore, if we notice that the effective refractive index $n_{\text {eff }}$ of the SPs on each of the cylinders depends on their radius [10], and that it increases by the narrowing of the cylinders, it is straightforward to conclude that the thinner the cone tip the higher the SPs refractive index. In this context both the real $\left(n{ }^{\prime}\right.$ eff $)$ and the imaginary ( $n$ "eff) part of $n_{\text {eff }}$ sensitively increase while the radius is decreasing (Figure 1b) [11].

If a SP is properly induced in the conical structure, it will propagate towards the tip experiencing an increase of the refractive index, thus slowing down while shrinking its effective wavelength since $\lambda_{\text {eff }}=\lambda_{0} / \mathrm{n}^{\prime}$ eff (Figure 1a).

However, it is not trivial to induce the adiabatic compression of a SP. In fact it has been shown that a TM0 mode within the structure is needed in order to obtain the phenomenon [12-14]. This means that the propagating plasmon needs a particular symmetry in order to be squeezed along a tapered structure. This requirement can be qualitatively understood in terms of charge distribution (Figure 1d,e,f,g): the TM0 mode expresses a field configuration requiring a net charge per section; thus this mode is unlikely to couple with outer media. If it is induced in a conical structure it leads to electric field concentration.
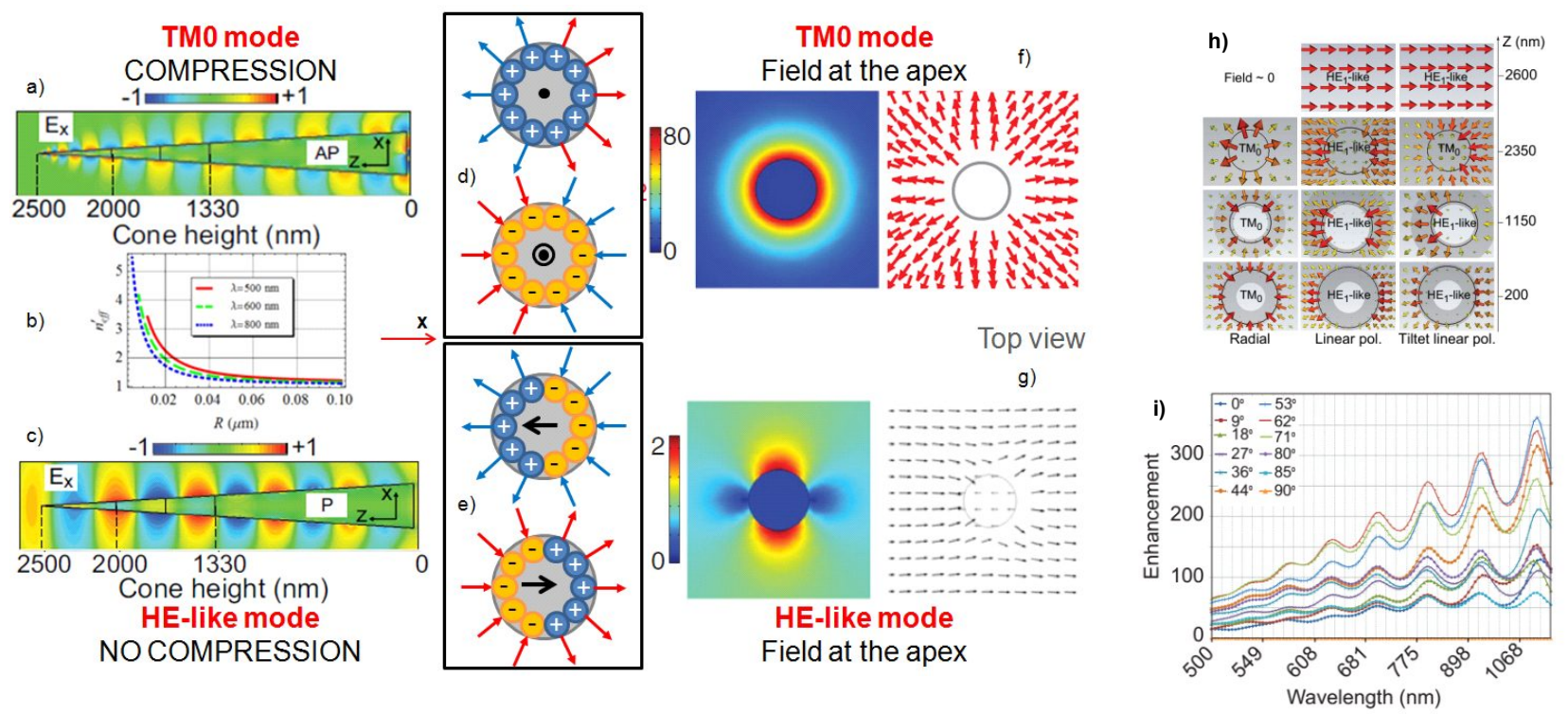

Figure 1: a) Electric field in the $\mathrm{x}$ direction for a SP propagating in a conical structure. The anti-phase (AP) configuration enables a TM0 mode satisfying the requirements for the adiabatic compression [12]. b) Dependence of the real part of the effective refractive index on the radius value of a metallic cylinder [5]. c) Electric field in the $\mathrm{x}$ direction for a SP propagating in a conical structure. The in-phase condition (P) does not allow adiabatic compression and field concentration at the tip [12]. d) Oscillating charge distribution for a given section of the cone in a TM0 mode. e) Oscillating charge distribution for a given section of the cone in a HE-like mode. f) Field configuration at the tip for a TM0 mode. g) Field configuration at the tip for a HE-like mode. h) Vectorial distribution of the electric field on cone sections for three configurations. Four values of $z$ (parallel to cone axis) are considered, $\lambda=514 \mathrm{~nm}$. i) Angle dependence of the electric field enhancement for wavelength range $(500-1200 \mathrm{~nm})$. The field was measured $10 \mathrm{~nm}$ off the cone tip.

As we can see in Figure 1d,e, at each section of the cone the charge distribution of a TM0 mode is associated with a nonzero divergence of the electric field inside the cone while it is zero outside (where there are no charges). Therefore, the coupling between the mode within the structure and a mode outside it is very unlikely to happen. The main consequence is the confinement of the SP on the surface of the metallic cone without any possibility of radiating, namely it is forced to travelling along the cone surface and concentrating while reaching its tip. On the other hand, a HE-like mode has a zero-value divergence of the electric field also inside the metallic structure. This characteristics determines a cut-off for the mode which allows it to leave the conical structure by its conversion into radiation once a minimum radius is reached. In this case no field concentration occurs as shown in Figure 1c.

One important issue in achieving adiabatic compression is thus the ability to induce a TM0 in the metallic tapered structure. Although the ideal excitation, for this purpose, would be a radial source [6] (since it has the field and phase 
configuration of a TM0 mode) its implementation presents some challenges from an experimental point of view. However, it has been shown that also a tilted linearly polarized EM wave can trigger the adiabatic compression, thus leading to sensitively simplified experimental setups $[12,14]$.

In Figure 1h we can observe how the TM0 electric field configuration within a section can be induced both by a radial source and a tilted linearly polarized EM wave allowing high field enhancement values (Figure 1i).

\section{SUPERHYDROPHOBIC SURFACES FOR ATTOMOLAR DETECTION}

In section 1.1 it was shown how to obtain very high electric fields in a nanometric volume. A direct application of the process can be found combining an AFM cantilever with a metallic cone grown at the end of it. Being able to induce a radial mode into the cone allows merging atomic force microscopy and Raman spectroscopy within the same set-up [15]. Owing to this technique, chemical mapping down to $7 \mathrm{~nm}$ was obtained [6].

However, when biomedical sensing is considered, a precise chemical mapping is just one of the key issues. In fact, efforts in reaching early diagnosis techniques require biomedical sensing device with the capability to detect low concentrated analytes dispersed in a solution [16]. Whereas Raman spectra enhanced by SPs adiabatic compression can, in principle, solely detect single/ few molecules owing to nanometric hot-spot volumes, low concentrated solutions together with diffusion limit forbid any practical application. In fact, to make this technique working target molecules need to be in contact with the tip of the conical structure which can be a few nanometers wide. In 2005 it was shown that the time required for the first ten molecules in a $1 \mathrm{fM}$ solution to accumulate in a $20 \mathrm{~nm}$ wide circle area is between 1 day and 1 month [17].

It is clear that this technique alone would poorly perform in such a context. In order to overcome the issue, superhydrophobic surfaces have been considered. Super-hydrophobicity refers to the phenomenon in which a drop on a surface adopts a contact angle greater than $150^{\circ}[18,19]$. Since the property is preserved for any dimension of the drop (prior to collapse) we can exploit evaporation to manage even ultra-low concentrated solution: while the solvent evaporates, the drop reduces its volume increasing the relative concentration of the analyte. The concept is expressed in Figure 2:
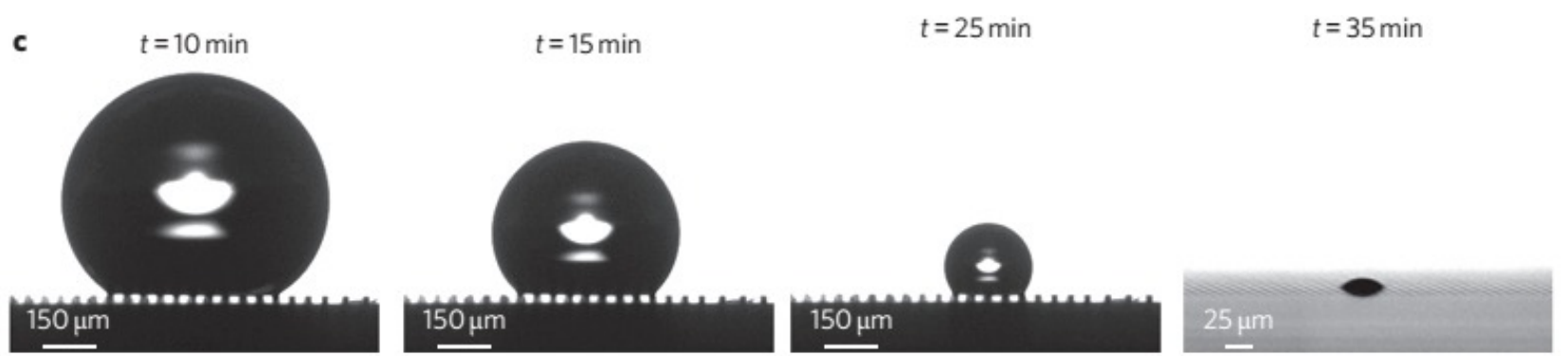

Figure 2: evaporation process for a solution drop on a super-hydrophobic surface. The initial diameter of the drop is $2 \mathrm{~mm}$ (not shown). Throughout the process no solute is left on the substrate. The drop slips on the surface while keeping its contact angle constant. The last image shows the drop after its collapsing [19].

A super-hydrophobic surface can be obtained through an array of micro-pillars [20]. By optimizing the pillars diameter and the array pitch it was possible to obtain a robust lattice on top of which evaporation could take place, namely squeezing of the analyte in a narrow volume despite its low initial concentration. Furthermore, by a prior symmetry breaking of the pillars pattern (for example by removing a pillar), the analyte tends to concentrate around the imperfection. In fact, it can be shown that an imperfection of the array induces an energetically favorable state in the area where the cavity is formed [19].

This phenomenon has consequences on the evaporation dynamics of the solution: the effect is that during the evaporation the drop is pulled towards the imperfection. As a result, after the drop collapse, the analyte is placed in the spot where the symmetry was broken. If the symmetry is broken by turning one micro-pillar into a conical structure, for example by tapering the structure with a Focused Ion Beam (FIB), it is possible to concentrate the molecules in a narrow region where high electric fields can be induced through surface plasmons adiabatic compression. Finally, in order to induce the 
coupling between the cone and the incident electromagnetic field, a properly designed grating was milled on one side of the structure (Figure 3).
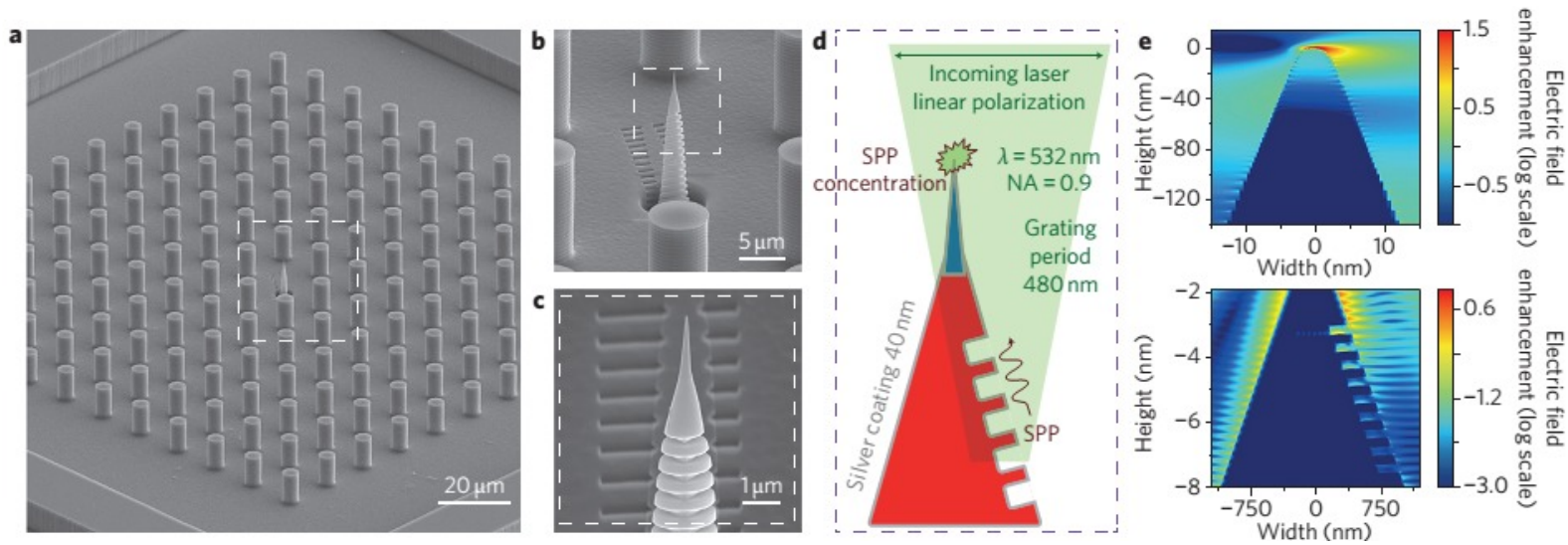

Figure 3: a) super-hydrophobic surface with the central pillar converted into a cone. b,c) Details of the central conical structure and grating. d) Measurement principle: Through a scanning procedure an optimal illumination/ detection of the grating/ sample is obtained. e) FDTD simulations of the electric field induced at the tip of the cone [19].

Owing to this technique a Raman enhancement of $1.5 \cdot 10^{6}$ was obtained allowing the detection of the vibrational modes of lysozyme (an enzyme of the immune system which acts by binding to certain bacteria molecules). The experiment considered a 160nl droplet containing $1 \mathrm{fM}$ lysozyme (about 100 molecules) deposited onto the surface using a microinjector system. After the evaporation lysozyme accumulated on the cone and Raman spectra were acquired showing chemical information about the protein (Figure 4.)
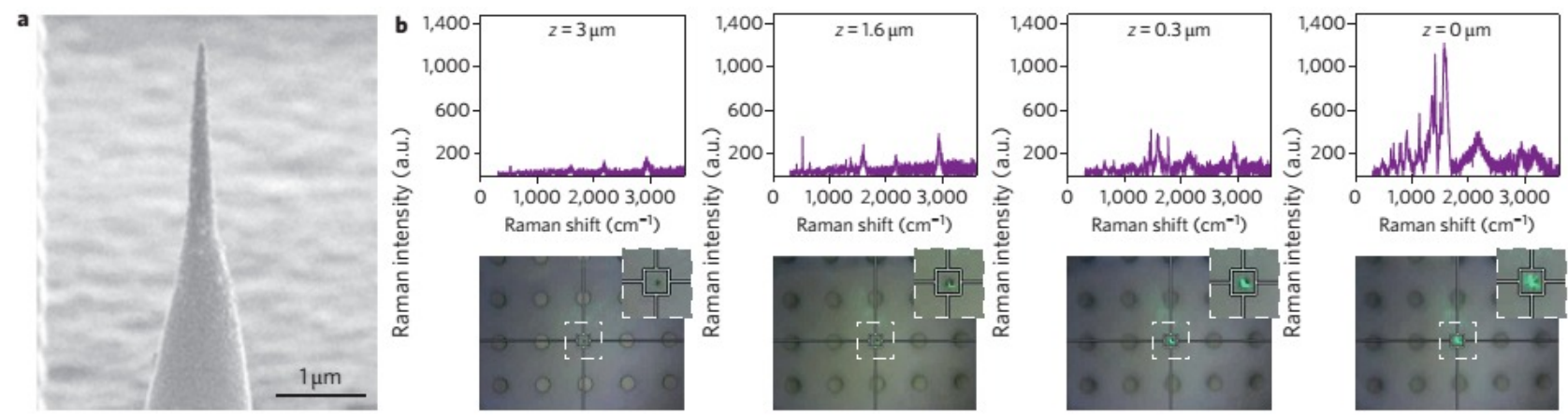

Figure 4: a) SEM image after lysozyme precipitation. b) Optical images and corresponding Raman spectra while scanning along the the optical axis.

With this approach it was possible to overcome, at the same time, diffraction and diffusion limits allowing few molecules detection in highly diluted solutions, paving the way to early detection of diseases where the onset of pathology is often due to a small number of molecules. 


\section{3D SERS DEVICES FOR SINGLE/ FEW MOLECULES DETECTION}

In section 2 it was shown how the combination of super-hydrophobic surfaces with the adiabatic compression phenomenon can lead to few molecules detection. The aforementioned technique allows indeed molecules detection on highly diluted solution but it has no sensitivity regarding the molecular species contained in the solution. After droplet evaporation all the analytes are accumulated near the tip and the acquired Raman (SERS) signal is actually a weighted average of the spectra of the single types of different molecules. This means that if the sample contains different kind of molecules it is not possible to discern them.

A step towards single/ few molecules detection can be represented by arrays of coupled nanostructures where a high number of electric field hot-spots are generated [21]. Therefore, at very low concentrations $(\sim 1 \mathrm{pM})$, a single/ few molecules per hot-spot are statistically accumulated.

The platform that was proposed in [22] consists of 3D nanostars dimers built on silicon pillars arranged in an array fabricated by means of electron beam lithography (EBL) and reactive ion etching (RIE). Although the top-down approach can be associated to high costs and it suffers of a number of constrains which limit the structures design, it also allows fabrication reproducibility, uniformity of the generated electric fields and a tight control over hot-spots positions and sizes.
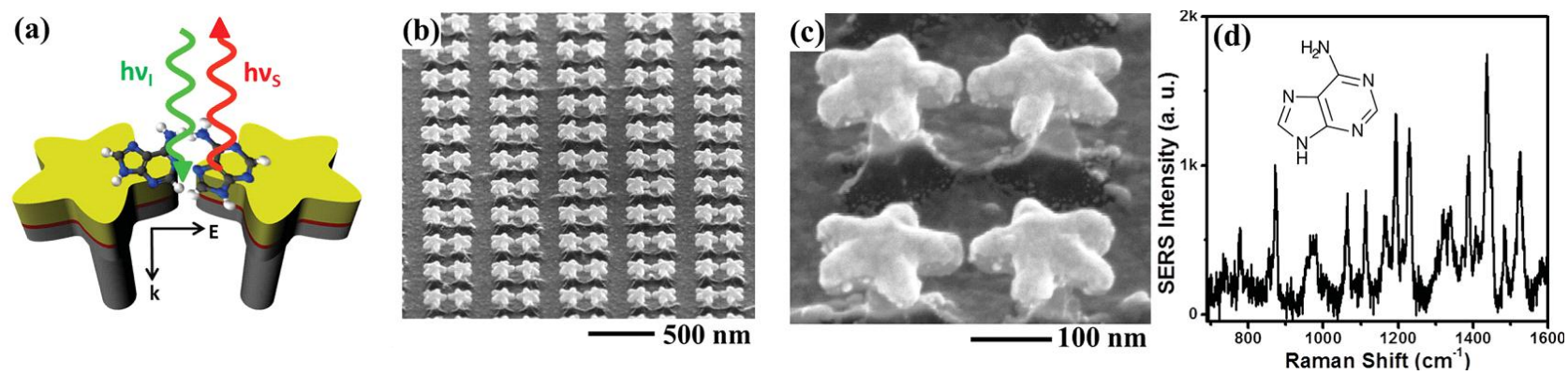

Figure 5: a) Working principle of SERS for the proposed geometry. b, c) SEM images of the dimers array. d) Raman spectrum of adenine chemisorbed from a $1 \mathrm{pM}$ solution.

In Figure 5a the working principle of the fabricated dimer is sketched. The peculiarity of the geometry relies on the narrow tips which ensure high electric fields. The elevated configuration allows on one hand an inter-particle distance below 10nm [23-25] therefore strongly increasing the dimer field enhancement and, on the other hand, it prevents the electric field to spread into the substrate. The optimized geometry has pillars $150 \mathrm{~nm}$ high with an inter-particle distance of $6 \mathrm{~nm}$. The testing of the device was accomplished by depositing $p$-MA molecules $(10 \mu \mathrm{M})$ resulting in a total SERS enhancement factor of $1.110^{7}$ (the Raman spectra were compared with a planar gold film). Sensitivity of the dimers has instead been investigated acquiring SERS spectra employing adenine molecules (1pM). Adenine is one of the two purine nucleobases which are part of the nucleotides in nucleic acids; its function is related to the stabilization of the nucleic acid by binding with thymine. In Figure 5d the corresponding Raman spectra are presented together with the chemical structure of adenine (see inset Figure 5d). In the figure many spectral features belonging to the molecule are shown, in particular the characteristic purine-ring breathing mode at $736 \mathrm{~cm}^{-1}$ and the $\mathrm{C}-\mathrm{N}$ stretching mode at $1319 \mathrm{~cm}^{-1}$ thus proving the high sensitivity of the technique. 


\section{ADIABATIC COMPRESSION FOR HOT ELECTRON NANOSCOPY}

In section 1.1 the adiabatic compression phenomenon was introduced and it was shown how electromagnetic waves can couple with metallic conical structures in order to realize surface plasmon polaritons. These quasi-particles would then propagate along the tapered geometry to concentrate at the tip end to generate a strong electric field. SPs are bounded mode decaying from the interface in both media (metal and surrounding dielectric) losing energy along the propagation. The energy loss is scattered in the environment as radiation by means of photons (surface roughness, tip-end emission) and into the metal in a multiple step process which involves photon-electron ( $p h-e)$, electron-electron $(e-e)$ and electronphonon $(e-p)$ interactions [26]. While $e-e$ and $e-p$ scattering processes eventually lead to power dissipation and consequent heating of the structure, ph-e interaction produces, at first, electron-hole pairs and thus "hot"-electrons [9]. These particles have an energy corresponding to the wavelength of the electromagnetic radiation inducing the surface plasmons. By considering the hot-electrons generated within the electrons mean free path ( $\sim 20 \mathrm{~nm}$ at optical frequencies) from the tip end, it possible to utilize the conical structure as a probe to investigate a semiconducting substrate (tip and substrate would form a Schottky barrier).

The proposed device [9] consists of an AFM customized set-up in contact mode with the sample, combined with laser sources at different wavelengths which can couple surface plasmons on the tip. This configuration allows for simultaneous topological and electronic investigation of the sample owing to atomic force microscopy and energetic hotelectrons overcoming the Schottky barrier.
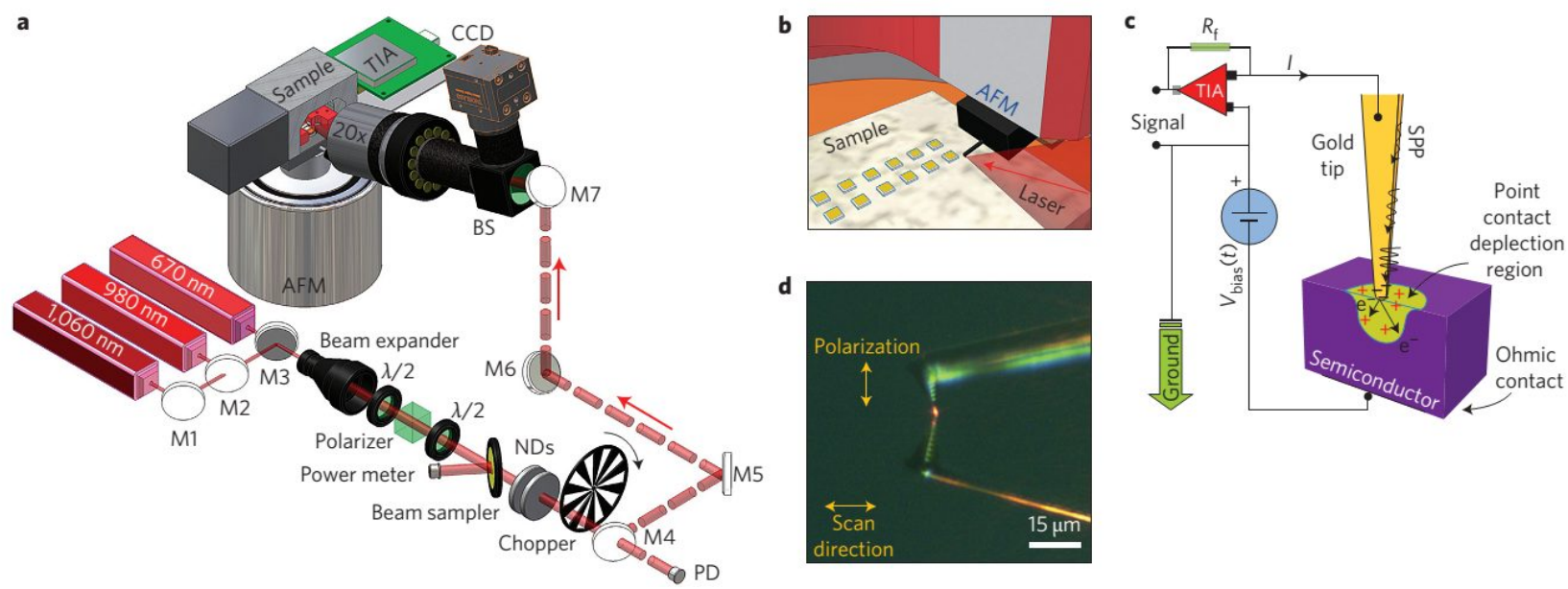

Figure 6: a) Schematic of the experimental set-up: one of the $10 \mathrm{uW}$ lasers impinges on the grating while amplitude-modulated with a chopper at $500 \mathrm{~Hz}$ and focused on the AFM tip. Additional elements, as indicated, were used to monitor (photodiode, PD) and manage the polarization, intensity (neutral density filters, NDs) and beam profile of the incident radiation. M1-M7 are mirrors; BS, beamsplitter. b) Sketch of the sample configuration. c) Basic circuit scheme: a transimpedance amplifier (TIA) FEMTO, gain $=10^{8}$, bandwidth $=50 \mathrm{kHz}$ performed the junction current amplification. An external bias voltage circuit was usedto polarize the junction, and a lock-in amplifier SR830 and a NI6366 data acquisition board were used to acquire and record the signals. d) A confocal CCD (charge-coupled device) allows real-time monitoring of the scanning and illumination states. The optical spot at the cone apex is a result of the radiative decay of the SPP when the device is illuminated by a laser with a wavelength of $670 \mathrm{~nm}$. One of the twin images is the reflection from a GaAs surface. [9]

The dominant effect of the device is an enhancement of the internal photoemission (IPE). Once coupled SPs are converted into hot electrons, these may overcome (provided they have enough energy and proper momentum) the Schottky barrier between the tip and semiconductor adjacent to it. As result they enter the depletion region of the semiconductor and relax to the lower states of the conduction band. These electrons can then be collected as photocurrent. Since the dynamic of the process is strongly dependent on the sample electronic characteristics, this technique represents a unique tool to study at the same time electronic and morphological properties of a semiconductor. The main advantage of this configuration is due to the high efficiency through which hot electrons are generated that reach $\sim 30 \%$ in the present system compared to $\sim 1 \%$ reported in previous experiments [9]. 

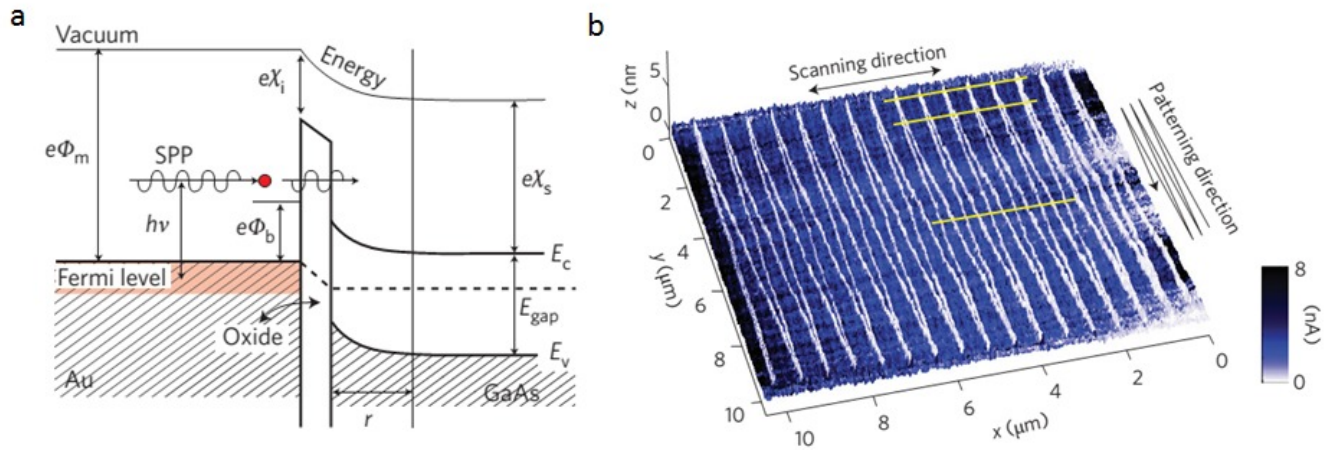

Figure 7: a) Energy band diagrams of the Au-GaAs junction where the oxide layer is present. b) Topography of the GaAs sample with $0.7 \mathrm{~nm}$ oxide patterning. b) Photocurrent imaging overlaid on three-dimensional topography, showing simultaneously the achieved current and topographic resolution.
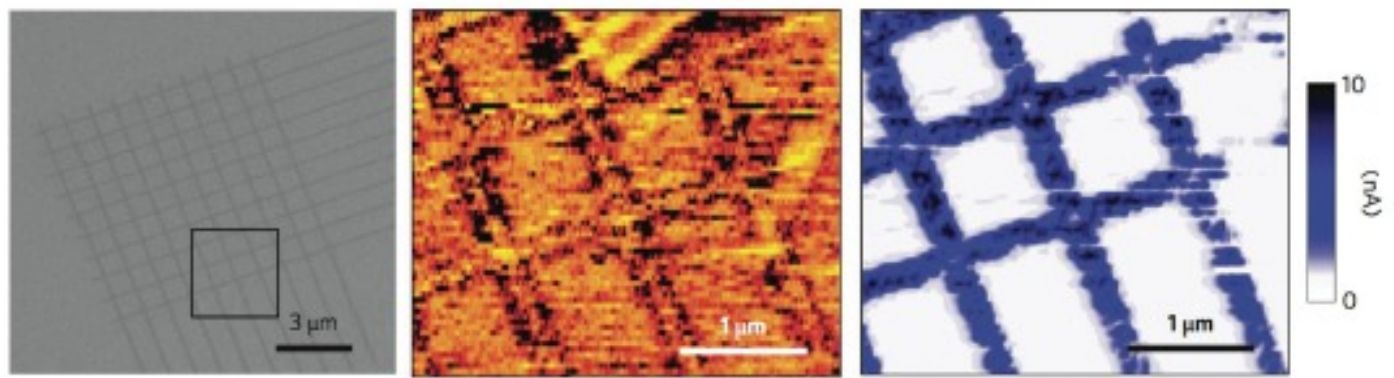

Figure 8: a) Energy band diagrams of the Au-GaAs junction where the oxide layer is present. b) Topography of the GaAs sample with $0.7 \mathrm{~nm}$ oxide patterning. b) Photocurrent imaging overlaid on three-dimensional topography, showing simultaneously the achieved current and topographic resolution.

The device was used to perform simultaneous topographic and photocurrent imaging of a GaAs layer on top of which a $0.7 \mathrm{~nm}$ oxide layer was patterned using a high field discharge technique [27]. The idea is that for a given wavelength $\left(\mathrm{E}_{\text {gap }}>h v>e \Phi_{\mathrm{b}}\right.$ ) the number of hot electrons overcoming the barrier between gold and the sample is dependent on the barrier height. The barrier is higher for Au-oxide-GaAs interface than for Au-GaAs as shown in Figure 7a. Therefore, we expect the collected photocurrent to be greater for the Au-GaAs as shown in Figure $7 \mathrm{~b}$ where low current (lighter regions) stripes are clearly visible along the lines where oxide was patterned with a resolution below $50 \mathrm{~nm}$.

In Fig. 8 is shown the topography (middle) and plasmonic hot-electron maps (generated at $980 \mathrm{~nm}$ laser excitation, right) acquired on a locally Ga ion-implanted GaAs substrate (SEM image, left). Clearly, the hot-electron map better discriminates the different regions with respect to the topography image.

It is important to underline that this experimental set-up can be further improved in terms of resolution and photocurrent efficiency by shrinking the radius of curvature of the nano-tip below $10 \mathrm{~nm}$. A smaller nano-tip would allow higher energy concentration, linear momentum exchange to hot-electrons and further confinement of the depleted region.

\section{REFERENCES}

[1] Barnes, W. L., Dereux, A., and Ebbesen, T. W., "Surface plasmon subwavelength optics," Nature, 424(6950), 824-830 (2003).

[2] Barnes, W. L., "Surface plasmon-polariton length scales: a route to sub-wavelength optics," Journal of Optics A-Pure and Applied Optics, 8(4), S87-S93 (2006).

[3] Das, G., Chirumamilla, M., Toma, A., et al., "Plasmon based biosensor for distinguishing different peptides mutation states," Sci. Rep., 3, (2013). 
[4] Mayer, S. A., [Plasmonics: Fundamentals and Applications] Springer, New York, NY 10013, USA(2007).

[5] Challener, D. S. A. W., [Modern Introduction to Surface Plasmons: Theory, Mathematica modeling and Applications] Cambridge University Press, USA(2010).

[6] De Angelis, F., Das, G., Candeloro, P. et al., "Nanoscale chemical mapping using three-dimensional adiabatic compression of surface plasmon polaritons," Nature Nanotech., 5(1), 67-72 (2010).

[7] Babadjanyan, A. J., Margaryan, N. L., and Nerkararyan, K. V., "Superfocusing of surface polaritons in the conical structure," J. of Appl. Phys., 87(8), 3785-3788 (2000).

[8] Stockman, M. I., "Nanofocusing of Optical Energy in Tapered Plasmonic Waveguides," Phys. Rev. Lett., 93(13), 137404 (2004).

[9] Giugni, A., Torre, B., Toma, A., et al., "Hot-electron nanoscopy using adiabatic compression of surface plasmons," Nature Nanotech., 8(11), 845-852 (2013).

[10] Song, J. F., Proietti Zaccaria, R., Dong, G., et al., "Evolution of modes in a metal-coated nano-fiber," Opt. Exp., 19(25), 25206-25221 (2011).

[11] Proietti Zaccaria, R., Alabastri, A., De Angelis, F., et al., "Fully analytical description of adiabatic compression in dissipative polaritonic structures," Phys. Rev. B, 86(3), 035410 (2012).

[12] Zaccaria, R. P., De Angelis, F., Toma, A., et al., "Surface plasmon polariton compression through radially and linearly polarized source,” Opt. Lett., 37(4), 545-547 (2012).

[13] Alabastri, A., Toma, A., Liberale, C., et al., "Interplay between electric and magnetic effect in adiabatic polaritonic systems," Opt. Express, 21(6), 7538-7548 (2013).

[14] Malerba, M., Alabastri, A., Cojoc, G., et al., "Optimization of surface plasmon polariton generation in a nanocone through linearly polarized laser beams," Microelectron. Eng., 97(0), 204-207 (2012).

[15] De Angelis, F., Zaccaria, R. P. , Francardi, M., et al., "Multi-scheme approach for efficient surface plasmon polariton generation in metallic conical tips on AFM-based cantilevers," Opt. Express, 19(22), 22268-22279 (2011).

[16] Razzari, L., Toma, A., Shalaby, M., et al., "Extremely large extinction efficiency and field enhancement in terahertz resonant dipole nanoantennas," Opt. Express, 19(27), 26088-26094 (2011).

[17] Sheehan, P. E. , and Whitman, L. J., "Detection Limits for Nanoscale Biosensors," Nano Letters, 5(4), 803-807 (2005).

[18] Sun, M. H., Luo, C. X., Xu, L. P. et al., “Artificial lotus leaf by nanocasting,” Langmuir, 21(19), 8978-8981 (2005).

[19] De Angelis, F., Gentile, F., Mecarini, F., et al., "Breaking the diffusion limit with super-hydrophobic delivery of molecules to plasmonic nanofocusing SERS structures," Nature Photonics, 5(11), 683-688 (2011).

[20] Shoji, S., Zaccaria, R., Sun, H. B., Kawata, S., "Multi-step multi-beam laser interference patterning of threedimensional photonic lattices," Opt. Exp., 14(6), 2309-2316 (2006).

[21] D'Andrea, C., Bochterle, J., Toma, A., et al., "Optical Nanoantennas for Multiband Surface-Enhanced Infrared and Raman Spectroscopy," ACS Nano, 7, 3522 (2013).

[22] Chirumamilla, M., Toma, A., Gopalakrishnan, A., et al., "3D Nanostar Dimers with a Sub-10-nm Gap for Single-/Few-Molecule Surface-Enhanced Raman Scattering,” Advanced Materials, n/a-n/a (2014).

[23] Cabrini, A., Barsotti, R. J., Carpentiero, A., et al., "Cross beam lithography (FIB+ EBL) and dip pen nanolithography for nanoparticle conductivity measurements," J. of Vac. Sci. Technol. B, 23, 2806-2810 (2005).

[24] De Angelis, F., Liberale, C., Coluccio, M. L., et al., "Emerging fabrication techniques for 3D nano-structuring in plasmonics and single molecule studies," Nanoscale, 3, 2689-2696 (2011).

[25] Neubrech, F., Weber, D., Katzmann, J., et al., "Infrared Optical Properties of Nanoantenna Dimers with Photochemically Narrowed Gaps in the 5 nm Regime," ACS Nano, 6, 7326-7332 (2012).

[26] Alabastri, A., Tuccio, A., Giugni, A., et al., "Molding of Plasmonic Resonances in Metallic Nanostructures: Dependence of the Non-Linear Electric Permittivity on System Size and Temperature," Materials, 6(11), 48794910 (2013).

[27] Lorenzoni, M., Giugni, A., and Torre, B., "Oxidative and carbonaceous patterning of Si surface in an organic media by scanning probe lithography,” Nanoscale Research Letters, 8(1), 75 (2013). 